\title{
Design of a robotized workstation making use of the integration of CAD models and Robotic Simulation software as way of pairing and comparing real and virtual environments
}

\author{
Karol Velíšek ${ }^{1}$, Radovan Holubek ${ }^{1, *}$, Daynier Rolando Delgado Sobrino ${ }^{1}$, Roman \\ Ružarovský ${ }^{1}$, and Nina Vetríková $^{1}$ \\ ${ }^{1}$ Slovak University of Technology in Bratislava, Faculty of Materials Science and Technology in \\ Trnava, Institute of Production Technologies, 91701 Trnava, Slovakia.
}

\begin{abstract}
Over the last years, there has been an increasing tendency and pressure on the faster implementation robotic devices and systems in manufacturing. Such transition involves several disciplines starting with the prototyping of CAD models itself. The paper addresses the creation of CAD models and is mainly aimed at their integration in a given simulation environment according to the conception and guidelines of Industry 4.0, where the part itself becomes the entity carrying most of the needed information at any time of a production process. The creation of such CAD models is key for the further and better customization of simulations. In other to better exemplify all this, the paper describes the whole process of "virtual to real life implementation" of a given robotized workplace needed to be developed at the Institute. The design of such robotized workplace included the use of an ABB IRB 120 robot and several other devices which were all designed, simulated and analyzed in a virtual environment before the final development and implementation. This paper helped demonstrating the importance of having exactly the same model (real and virtual) with respect to the success of the offline simulations.
\end{abstract}

\section{Introduction}

Since the very beginning and course of the 3rd Industrial Revolution, the use of PLCs, Robotics and automation in general have played an outstanding role in the development of manufacturing. They have all become an irreplaceable asset that has survived all time boundaries and keep being of vital importance today. Robotics and automation have not only helped increasing productivity and taken manufacturing to another level, but they have also had a significant role in cost reduction and have contributed to make work safer than ever. However, everything has not been that easy, together with all these benefits companies have also seen themselves immersed in the constant training of their workers

\footnotetext{
*Corresponding author: radovan.holubek@stuba.sk
} 
and redesign of their production plants in order to really meet all the possibilities robotization and automation offer.

All what is mentioned above has not only impacted production giants but also small medium enterprises and educational institutions like the Institute of Production Technologies itself.

This institution shares a great responsibility in the training of national specialist and students because of its strategic positioning in the canter and surroundings of the bigger manufacturing hubs of the country.

It has been proved that the most effective way to deal with new technological challenges and new tendencies, e.g. Industry 4.0 nowadays, is the training and preparation of the future workforce since the very base.

This specially applies not only to Robotics and Automation but also to their linkage to CAD designs software which are all very important and complex disciplines whose the Slovak industry so badly asks for, needs and the present papers moves around.

Being aware of all above mentioned elements, the present paper introduces the design of a robotized workstation.

Such design has been conceived from the very CAD design and has been then simulated offline in RobotStudio before the real implementation, this has offered the possibilities of pairing and comparing both the virtual and real environments and somehow qualitative study the effectiveness of the models before the real implementation in one of the teaching laboratories of the institute.

The rest of this paper is divided in: Section 2: Design of the workstation in CATIA V5, section 3: Modelling of the workstation in the RobotStudio environment, Section 4: Construction of the designed workstation and analysis of the virtual and real environments, Section 5: Conclusions and further research, Section 6: Acknowledgement and finally a section of references.

\section{Design of the workstation in Catia V5}

This section shows the chronological steps in the creation of the workstation. The whole conception started from considering the robot type (ABB IRB 120), the parts to manipulate and the pallets to be used.

As for the robot type it was mainly useful to take into account its weight, dimensions, movements angles among a few other technical information available at the manufacturer' website, see [1].

Based on the previous information, the base frame of the workstation was given the dimensions shown below. It was important to create a stable frame able to deal with the full-speed movements and acceleration of the robot in the worst case scenarios.

Most of the profiles used were made of aluminum and can be found in [2].

- Dimensions of the base frame: 1300x1000x1000 mm, it was made of 40x40 mm aluminum profiles.

As it can be also seen in Fig. 1, a wooden table was inserted into the design so as to secure a much better stiffness of it.

At the same time it will be also used for placing the robot controller, the wooden material does better avoids shakings who may well have a negative effect on the structure and integrity of the Controller. 


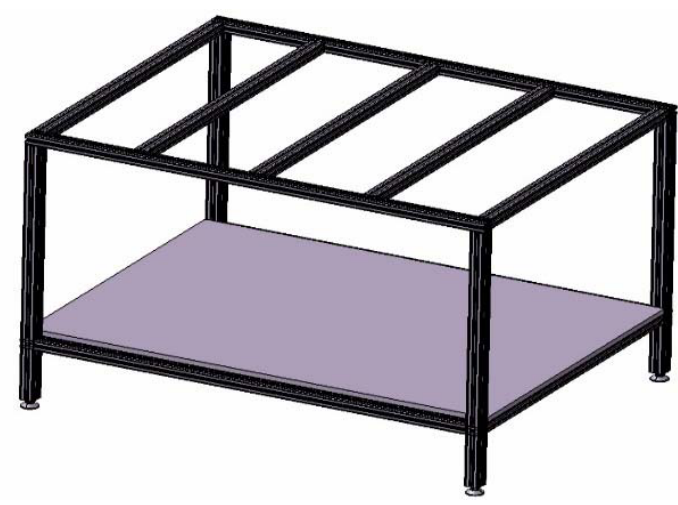

Fig. 1. Design of the base frame.

In a similar way the work table was also created, see Fig. 2.

- Dimensions of the profiles in the work table: 1400x1200 mm. The profiles were of 40x80 $\mathrm{mm}$ and were cut (in length) at $1400 \mathrm{~mm}$, while 15 of those profiles were fixed together giving the needed width of $1200 \mathrm{~mm}$.

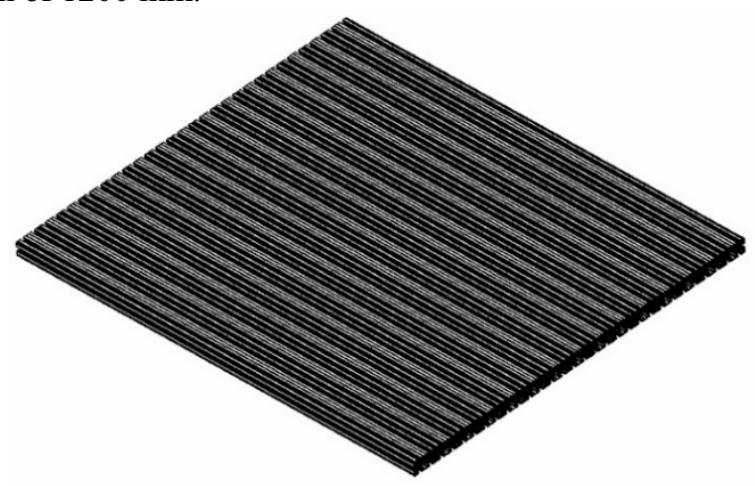

Fig. 2. Design of the work table.

A better fixation of the robot on the above shown table is to be achieved by using the steel base plate under its base.

This has been also design as shown in Fig. 3 .

Dimensions of the steel plate: 370x400x10 mm.

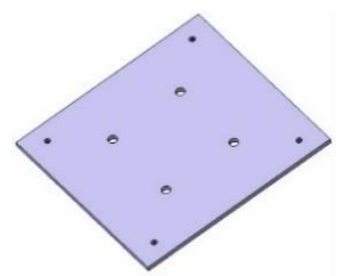

Fig. 3. Design of the steel base plate for the base of the robot.

Fig. 4 shows all of the previous element together in what would be the final workstation, still it does not include a series of safety elements [3]. 


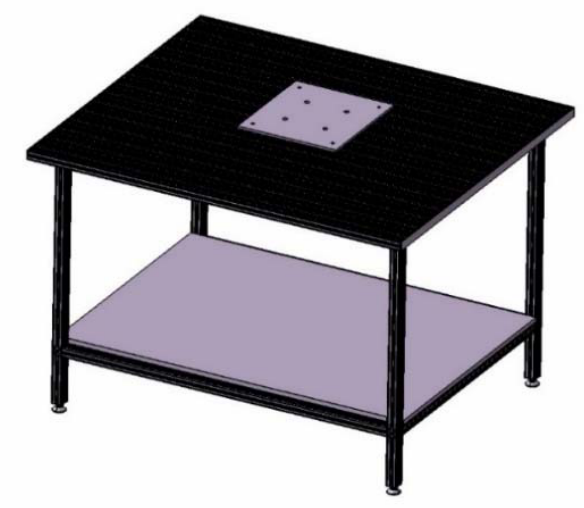

Fig. 4. Designed workstation without safety elements.

As for fulfilling some of the safety regulations of the robotized workstation to be located at a student's laboratory, the following safety barriers were also designed and added to the surroundings of the robot, see Fig. 5.

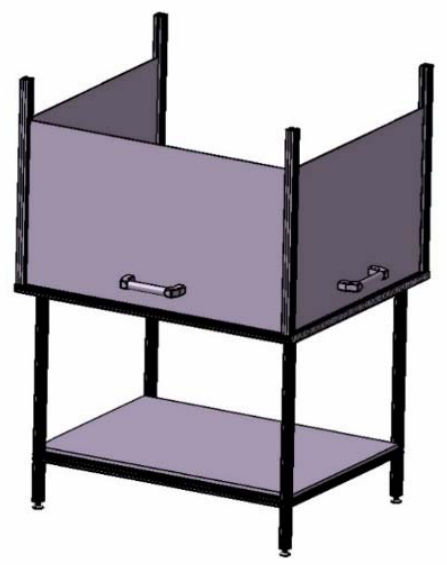

Fig. 5. Designed workstation with safety barriers.

\section{Modelling of the workstation in the RobotStudio environment}

In this section, all of the previous CAD models have been imported into the RobotStudio environment. Despite existing many options for file conversion, in this case, the STEP files coming from CATIA V5 were converted to SAT files using a CAD exchanger tool. With all this, the main idea was to look for the better layout configurations and prove the kinematics and reach of the robot to execute given tasks which was key prior to the definite construction of the workstation [4]. The following Fig. 6 shows the virtual environment of the workstation while executing a given operation in the student's laboratory of the institute. Based on this off-line analyses and simulations, the final workstation is to be finally constructed and compared [5]. Notice that after being all file types imported, it was necessary to place the robot in the "right "position on the previously designed steel base plate, for this it was necessary to make an analysis of the reach of the robot and its kinematics. 
Together with all this, several targets were created off-line so as to verify the positioning and visualize the functioning of the robot through simulation.

From all this process, students will contribute and continue to develop an existing database of CAD and SAT files that will be made available once appropriated and will have a positive impact on the teaching process $[6,7]$.

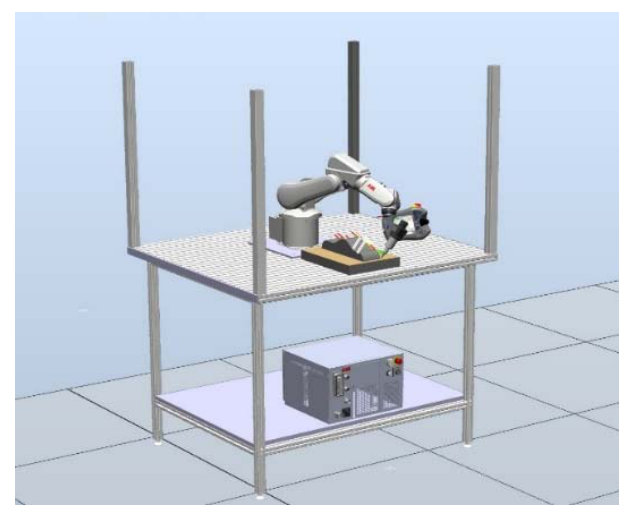

Fig. 6. Designed workstation in the RobotStudio environment at an institute's laboratory.

\section{Construction of the designed workstation and analysis of the virtual and real environments}

Taking into account all of the design elements mentioned above, also the off-line simulations and analyses made in RobotStudio, the physical version of the designed workstation is shown next in Fig. 7.

Notice that, as somehow already mentioned before, this is an educational workstation that besides serving research purposes leans mainly towards an enhancement in the teaching process in the laboratories of the faculty.

Within this workstation students are able to simultaneously play off-line simulations and execute those same movements in the real station, we can also make the students send their assignments via Ethernet and directly see their results in the workstation and in a similar way input their off-line programs into the USB ports of the robot controller [8].

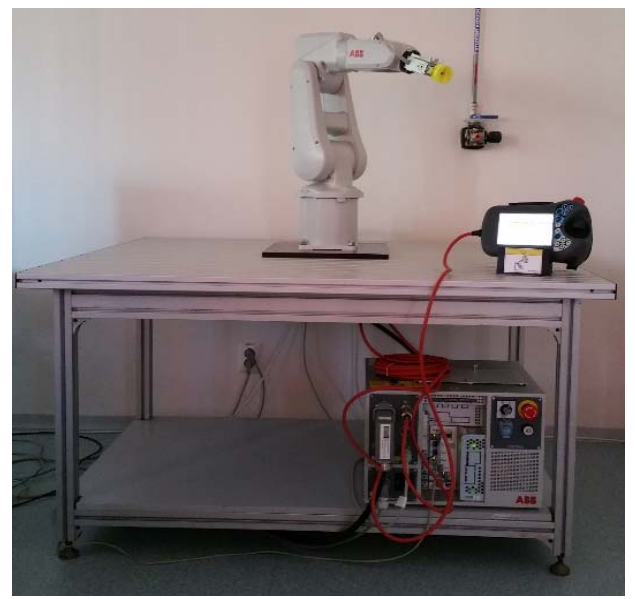

Fig. 7. Constructed workstation located at an institute' laboratory. 


\section{Conclusions and further research}

The present paper presented in a synthetized way the design, off-line simulation, tests and construction of a robotized workstation at one of the laboratories of the Institute of Production Technologies.

The whole process started with the CAD designs of the components of the workstation, then these were imported, simulated and tested off-line in the RobotStudio software. Once positioning, reach and other issues were analyzed the modelled workstation was constructed and finally compared with the models by executing the same movements created off-line.

The whole idea will positively impact the teaching process since it allows students to create off-line models and then simultaneously compare them with the real workstation. All this will also contribute to the creation of an educational database of files that will be used for exercises and much more within the teaching activities.

Further research is to encompass the construction and inclusion of many other devices and elements pertaining to the same station which have been already modelled and taken into account for this initial conception.

This work was supported by KEGA 027STU-4/2014: Establishment virtual laboratory of robotics and manipulation techniques.

\section{References}

1. ABB, Technical data for the IRB 120 industrial robot. Available from: http://new.abb.com/products/robotics/industrial-robots/irb-120/irb-120-data (2016)

2. FM Systeme Kompatible Profilreihen \& System-Engineering, Germany (2013)

3. J. Johnson, M. Kwoka, H. Houayek, I. Walker, K. Green, Design, construction, and testing of a novel robotic workstation. In Proceedings fourth international conference on computational intelligence, robotics, and autonomous systems (CIRAS). Palmerston North, New Zealand, 155-160, (2007)

4. P. Neto, J.N. Pires, A.P. Moreira, CAD-based off-line robot programming. Robotics Automation and Mechatronics (RAM), IEEE Conference, 516-521, (2010)

5. J. Zhang, X. Fang, L. Qi, Robot. Comput. Integr. Manuf., 38, 9-15 (2016)

6. S. Hao Deng., Z. Hua Cai., D. Dan Fang., H. Lin Liao., G. Montavon, Surface and Coatings Technology, 206, 19-20, 3875-3882 (2012)

7. R. Holubek, D.R. Delgado Sobrino, P. Koštál, R. Ružarovský, Applied Mechanics and Materials: Novel Trends in Production Devices and Systems II, 693, 62-67, (2014)

8. R. Holubek, K. Velíšek, M. Šimúnová, N. Vetríková, D. R. Delgado Sobrino: On the possibilities and the advantages of robot off-line programming: Teaching of off-line programming methods as a tool to further industrial implementation utilization. In 3rd International conference on Advances in mechanical, aeronautical and production techniques - MAPT. 1. Issue. New York: Institute of Research Engineers and Doctors, (2015) 